\title{
Potential-position Method for Human Organization Relationships
}

\author{
Weiliang Cheng \\ School of Energy, Power and Mechanical Engineering, North China Electric Power University, Beijing, China \\ cwl@ncepu.edu.cn
}

\begin{abstract}
It is not the direction and ultimate goal of today society that monopole effect of personal heroism are pursued unilaterally and partially, and the realization of human-maximumeffect strategy are the unchangeable truth consciously or unconsciously. Therefore, it has the urgency sense and guideline that the optimization potential-position principle in human effects is presented. The potential can be taken as the base concept to describe human organization relationships as well as responding potential description rule in different relationships. And then the optimization potential-position principle is obtained. No matter what the human system is big or not in the human organization relationship, the potential-position principle can be used to increase human systematic effect entirely. In future developed society in information and calculation technology, the potential-position principle may have more and more important significance.
\end{abstract}

Keywords: management science; human; system optimization; potential-position principle

\section{I . Introduction}

All the systematic effect decides the entire organization's living or death, however, all-round improvement of overall effect in the system can't be realized only by means of some heroic figure. The common wish about entire human society development is the optimization and improvement of overall effect[1-4]. In the simple communication among persons, and frequently in the personal relation with some crisis once in a while, and in our departments and enterprises, the size and range of different personal "radiative force" are not same. How to harmonize and improve the synthetical effect of entire human organization relationship to avoid the breakdown of the entire system, is very important to care for very much, and wait for urgently the problem studied, analysed and solved.

\section{II . Human Relationships in Systems}

The human organization relation is an interaction form in various activities, different levels and different effects[5-6]. People, who have certain organization relations and realize the certain mission, are composed of a system. The state of human organization relation is decided by personal position of different individuals in human system. In the realistic society, there are all kinds of person and environment, as a result, there are also different systems which realize the respective mission, for example, some large systems and small ones and so on. In these systems, some are obtained with some provisional relations. After their mission is completed, this relation has also been removed. Others are the kinds of organization relation that are fixed in the long term and consisted of some certain systems, but these systems are relatively stable, having ceaseless renewal and perfect inherent requirement to maintain the systematic long-term development.

Within certain time and space range, an ecosystem is a whole that consists of biological community and its environment. The ecosystem has certain size and structure, and every member gets in touch with each by means of energy flow, substance circulation and information delivery, and every member influencing, depending on each other, and furthermore form the compound body that has a function of self-organization and the self-adjustment. Persons have the biological attribute, but also have the language and thinking function, which are higher than ordinary living things. There are some ecosystems that take persons as a domination, which are called manpower compound ecosystem. In the ecosystems, other living things already lose the ability of self-regulating and controlling and self-recovering. At this time, under only human's active participation, renewal and substance circulation of other living things may be order in process. Considering only the system, which consists of people, the person relations are the sum of all kinds of the social relations, the system at this time is also called a social system. The person in the social system is a group in various organization relationships, and this decides that the system, which people get along with, is a kind of organization system. The persons in a organization system are affected by other persons in this system. Moreover, every personal effect is different. Therefore this implies that the same person plays a different role in different organization relationships, and different person also plays a different role in the different hierarchy of the same organization relationship[7-8]. How to rationally rearrange the effect of the organization relationship in order to improve the systematic synthetic effect as far as possible, is related to the key whether the system can be long and flourish or not.

\section{III . The Effect about the Personals}

Since each person plays a different role and have a different effect in the social system of self-organization relationship, their positions are also different in this organization system. In the organization system, the role and effect can be described with the potential. This potential contains two aspects. Firstly, there are the inherent factors of individual such as ability, spirit and inherent personal charm and so on; secondly, there is unique advantage including substance and self-confidence that the environment by accidental or inevitable cause gives him. For example, for a student group, some qualities are comparatively good and form the fairly good image easily, as a result, they 
conveniently hold opportunity to win a chance. Once these students have a little of conditions, their qualities of person leader is easy to take form, and it is easy to be preponderant cycles. And thus the "potential" of these students are comparatively big. However there are other students who doesn't strive for a chance very actively, and they have a comparatively strong mentality following the majority. And the starting point potential of the individual has an average standard. Even thought they had big potentials, they didn't express their merits because these potentials have a higher external threshold. Therefore these persons "being frustrated for all one's talent " also can only blame him self. And still have less students, because of congenital cause, the internal factors of them are relatively poor, and furthermore they have less confidence, and do not dare seek for the opportunity exceeding oneself much more, and their potentials are naturally comparatively lower.

Any man can't be perfect, and any gold also can't be pure. "Dark side and sunny side" are counterbalance in the world, and are able to counteract each other. The more sunlight and gorgeous exuberance of all things on earth are, the fainter it looks behind big trees and the buildings. The outstanding merit of one person is, the more his shortcoming hided is, and this like " the more loftier his stature is, the more bigger his projection in the sunlight is ". This can only explain that the time used is especially much more in the aspect of acquisition of merits, and the chances of the practice are especially more, therefore they are more outstanding than others. On the contrary, the time is few, and the experience and chances are fewer, thus their "defects" is bigger. And this is the reason why somebody is only discovered merit but not found his shortcomings. Usually, he is good at holding the aspect of merits in front of everybody, and it can not explain that he does not have shortcomings. At the same time, in different persons of the social system, their potentials also exist in the relations of unity of opposites by the size and environment. After the system is formed primary, the individual potential among them also appear relatively, which this potential is built synthetically according to its own factors and environmental factors. In the hierarchy construction of the potential, he is to relatively low relative to "higher authority". And he is relatively higher relative to the lower level. In other words, it shows that the potential has its space property. And the potential also has its temporality at the same time in the system. The potential is dynamic, and the individual potential varies with the time and space. After having formed individual potential in the social system, the position also has come into being in the system. When the individual potential is bigger in the social system, the corresponding potential is also comparatively high, that is to say, the bigger the individual effect and function are, the higher its position is in systematic hierarchy of society, and this is the potential-position principle.

\section{IV . Potential-position Optimization Method}

After discussing the potential and position in human organization relationship, it is necessary to study how to raise the systemic maximal effect. A social system is just like an electricity system, a physical system or an energy system etc, and it has a common property, namely, all of them are the networks that consist of Individual and the structural relation of the connection. However, the nodes in the network are, namely, individuals in the system. The concrete size of individual potential is different in the social system, and the position of the hierarchical structure in the organization relationship is also different. In the hierarchical structure that consists of nodes, the difference of its position also indirectly shows the size of its potential. Under the ordinary circumstances, the region of having high potential part in the social system is comparatively big, and its value exceeds the average value of all individual's potential in the system, whereas most of individual potentials are close to the average value of all the potentials. Extremely, a part of individual potential is comparatively fewer, namely low potential part. Because of congenital and other peculiar causes, they are lower than average value greatly. The distribution law of its "potential" in the system commonly show in Figure 1 as follows:

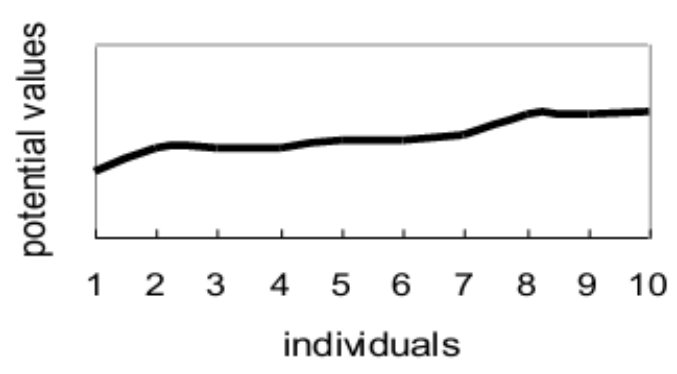

Fig.1 The distribution law of all potentials in the social system

Seen from this structural relation in Figure 1, the horizontal part shows that because most of the individual potentials are restricted by social civilization and the substance assumption standard at that time, the expressed potentials are close, and the major in the system generally represents the status of the development of society at that time. The impact of potential interior factors is to be decisive, such as human's initiative and the ability of grasping opportunity, but the premise that this decisive effect is played incisively is the friendly coordination of the external factors.

If the mathematical expectation value $f$ of the potentials ( namely average value in Equation (1) ) is much bigger, its potential value belongs to a high level, and the systematic stability is very good, and the developmental and perfect ability is higher.

$$
p=\sum_{k=1}^{n} \frac{\mu_{k}}{n}
$$

Where $K$ represents concrete individuals in the system, $\mathrm{n}$ is the sum of a systemic individuals, $u_{k}$ is a individual potential value of the system.

The smaller the square difference $f(p)$ of the potential in the system is, the bigger the systematic overall effect is, and the more sufficient the latent energy of the individual potential 
in the system are expressed. Then this is the optimization potential-position principle of human organization relationships in the social system. The form of the first-step mathematical description is as follows:

$$
f(p)=\min \sum_{k=1}^{n}\left(\mu_{k}-p\right)^{2}
$$

With the society development forward, productivity level and human's civilized degree improve unceasingly, and average level of the potential in the social system improves unceasingly. At the same time, the individual square difference of the potential in the system is also more and more small obviously, namely, the degree that the individual potential mean value in the system is more and more small. However, for the area of two poles of the potential in the system, namely, the part of the higher potential and the lower potential will be close to the mean value in the curve. In other words, with overall degree of human civilization improvement, the difference between potentials is decreasing. At this time, the contribution of the interior factors of the potentials is bigger and bigger, while the effect of the external factors of the potentials is smaller and smaller. At this time, the stable operation of the social system already depends on civilized degree of human intensely, but the punishment effect of laws and regulations is weak gradually. Human use the laws and regulations as the most fundamental of the acting standard and criterion. At this time, the entire future society and their own subsystems already displays flourishing and highly-free individual development state, and the times of individualistic heroism coming forth already elapse, and human are already in relatively gentle, equal, exoteric, comprehensive and selfgiving ideal state.

\section{V . Conclusions}

The individual potential is different in a social system. In the organization relationship, the role and effect of individual are different. In a system the potentials of individuals are divided into three parts, the first part is high potential, and the second part is average value, and the third part is low potential. The first part of the high potentials represents the leaderships in the system, and has the important guidance and determinative action, and the second part of the average value is the important strength which makes the system stable, and the third part of the low potentials has the serious hindrance and the negative influence in the advance of the overall system, which is the key to be solved for the promotion of the overall system strength. With the society developing, the level of mean value of the potential in the system rises gradually, and the degree of individual potential diverging mean value is smaller and smaller. At the same time, the effects of the interior factors of the potential are bigger and bigger, and while external factors are in smaller position.

\section{References}

[1] C Poole. "Conjecture and counterplea: The increasement of science knowledge," Shanghai: Shanghai Translation Press, 1986.

[2] Cai Xiaoming and Shang Yuchang. "Primary biology," Beijing: Peking University Press, 1995.

[3] Wang Qingzhao, Cheng Weiliang and Wang Jianxuan. "The effect of the exergy in the ecosystem," Modern Electric Power, vol. 14, no.1, pp. 20-25, 1997.

[4] Moran, P. "Structural vs. Relational Embeddedness: Social Capital and Managerial Performance," Strategic Management Journal, vol. 26, no. 12, pp. 48-57, 2005.

[5] Garcia, Carmen. "'Cuente conmigo': The expression of sympathy by Peruvian Spanish speakers," Journal of Pragmatics, vol. 42, no.2, pp. 408425, 2010.

[6] Subramaniam, M, YOundt, M. A. "The influence of intellectual capital on the types of innovative capabilities," Academy of Management journal, no.3, pp.450-463, 2005.

[7] Tirta, N, M. "Creating relational rents: The effect of business groups on affiliated firms, performance indonesia," Asia Pacific J Manage, no.23, pp.537-557, 2006.

[8] Rhona E J. "The role of focal suppliers in strategic networks for internationalisation," Journal of Fashion Marketing and Management, vol. 11,no.1, pp. 135, 2007. 